\title{
The 2012 IUJ impact factor: another step forward?
}

\author{
Paul Riss $\cdot$ Peter L. Dwyer
}

Published online: 27 July 2013

(C) The International Urogynecological Association 2013

In June 2013 the impact factors for 2012 were released in the Journal Citation Reports (JCR), an annual publication by the Healthcare \& Science Division of Thomson Reuters [1]. The 2012 impact factor for the International Urogynecology Journal (IUJ) was 2.169, an increase of $18 \%$ over the previous impact factor. While this increase is very welcome, the question remains: What does it mean, and where do we go from here?

The English word "impact" has various meanings but in the context of the impact factor of a scientific journal it means the average number of times an article from this journal is cited. The calculation of the impact factor is almost trivial: total number of citations (over a 1-year period) of items (articles) published in the preceding two calendar years, divided by the total number of citable items (original articles, reviews and case reports, but not usually editorials, letters to the editor, etc.) published in those 2 years. The total number of citable items published in the IUJ in 2010 and 2011 was 461, while the total number of citations in 2012 to items published in 2011 and 2010 was 1,000; the calculation, therefore, was $1,000 / 461=2.169$. One can also calculate a 5 -year impact factor (in the case of the IUJ 2.049), and the impact factor can be used to rank journals in a given field (the IUJ currently ranks 22/77 in Obstetrics and Gynecology and 26/73 in Urology and Nephrology).

One disadvantage of the impact factor is that although it measures how much (relative) attention a journal gets, it does

\footnotetext{
P. Riss $(\square)$

Medical University of Vienna, Anna Frauer Gasse 2, 1180 Vienna, Austria

e-mail: paul.riss@gmail.com

P. L. Dwyer

Mercy Hospital for Women, University of Melbourne, Melbourne, Australia

e-mail: pdwyer@connexus.net.au
}

not say anything about the impact on the scientific community of an individual article. It is therefore a little disingenuous when individual authors or institutions sum the impact factors of the journals in which their work has been published as a measure of the quality of their work. For this reason other metrics have been developed which do not look at the journal but rather at the attention an article gets by counting the number of times an article is cited (for example the h-index [2]). Some funding bodies, such as the Deutsche Forschungsgemeinschaft (German Research Foundation), have decided to ignore the impact factor and evaluate articles when making decisions regarding funding or appointments [3].

Nevertheless, we believe that the impact factor will stay with us for the foreseeable future, and we will work to further increase it for the IUJ. The best way to do this is to publish high-quality research papers and good reviews. The latter tend to be highly cited, but our aim will always be to provide our readers with a mix of articles which they find interesting and relevant to their practice. The impact factor can be influenced by encouraging self-citation or coercive citation practices, and by highly selective inclusion of manuscripts. We will avoid these practices and produce a journal of high quality and interest to our readership which also allows as many authors as possible to present their research and thoughts to an international audience.

Publishing is a rapidly changing world where technology and the ways readers acquire information are in constant flux. We suspect most articles nowadays are read electronically, although our surveys tell us that many still prefer the printed page. One video article published in the past few months has had more than 1,000 downloads. In the future, individual authors will probably make more use of social media specifically designed for the scientific community. ResearchGate is such a software platform where authors can post their profile, see what their peers are publishing, and learn how often and by whom their own work is downloaded or cited [4]. 
We appreciate the increase of the impact factor for the IUJ, and want to thank our authors for publishing their best work in the journal, our reviewers for their expert evaluation of manuscripts, and our readers for their continuing loyalty and support. The impact factor is one metric indicating that we are on the right track, but we will always continue to look for new ways to make the IUJ — the Blue Journal — as attractive as possible for readers, clinicians and researchers all over the world.

\section{References}

1. http://thomsonreuters.com/articles/2013/introducing-the-2013-editionjournal-citation-reports. Accessed 13 July 2013

2. http://en.wikipedia.org/wiki/H-index. Accessed 13 July 2013

3. http://www.dfg.de/en/service/press/press_releases/2010/pressemitteilung nr_07/. Accessed 13 July 2013

4. http://www.researchgate.net/. Accessed 13 July 2013 